\title{
Numerical simulation of fibrous biomaterials with randomly distributed fiber network structure
}

\author{
Tao Jin • Ilinca Stanciulescu
}

Received: date / Accepted: date

Original source of publication: https://link.springer.com/article/10.1007/s10237-015-0725-6

The final publication is available at link.springer.com.

Please cite as:

Tao Jin and Ilinca Stanciulescu (2016). Numerical simulation of fibrous biomaterials with randomly distributed fiber network structure. Biomechanics and Modeling in Mechanobiology, Vol. 15, No. 4, 817 - 830.

\begin{abstract}
This paper presents a computational framework to simulate the mechanical behavior of fibrous biomaterials with randomly distributed fiber networks. A random walk algorithm is implemented to generate the synthetic fiber network in $2 \mathrm{D}$ used in simulations. The embedded fiber approach is then adopted to model the fibers as embedded truss elements in the ground matrix, which is essentially equivalent to the affine fiber kinematics. The fiber-matrix interaction is partially considered in the sense that the two material components deform together, but no relative movement is considered. A variational approach is carried out to derive the element residual and stiffness matrices for finite element method (FEM), in which material and geometric nonlinearities are both included. Using a data structure proposed to record the network geometric information, the fiber network is directly incorporated into the FEM simulation without significantly increasing the computational cost. A mesh sensitivity analysis is conducted to show the influence of mesh size on various simulation results. The proposed method can be easily combined with Monte Carlo (MC) simulations to include the influence of the stochastic nature of the network and capture the material behavior in an average sense.
\end{abstract}

Tao Jin

Department of Civil and Environmental Engineering,

Rice University, Houston, TX 77005, USA

Ilinca Stanciulescu

Department of Civil and Environmental Engineering,

Rice University, Houston, TX 77005, USA

E-mail: ilinca.s@rice.edu
The computational framework proposed in this work goes midway between homogenizing the fiber network into the surrounding matrix and accounting for the fully coupled fiber-matrix interaction at the segment length scale, and can be used to study the connection between the microscopic structure and the macro-mechanical behavior of fibrous biomaterials with a reasonable computational cost.

Keywords Fibrous biomaterial · Finite element method $\cdot$ Monte Carlo simulation

\section{Introduction}

Many fibrous biomaterials, such as ligament and skin, can be categorized as fiber-reinforced materials and have randomly distributed fiber network structures at the microscopic scale (Stylianopoulos and Barocas 2007; Zhang et al. 2013a; D'Amore et al. 2010, 2014). These fiber networks can undergo large deformations and exhibit significant nonlinear behavior (Stella and Sacks 2007, Stella et al. 2010; Liu et al. 2013). The topology of the fiber network at the microscopic level can largely influence the material macro-mechanical behavior (Roeder et al. 2002, Chandran et al. 2008; Lake et al. 2012), for example, making the material isotropic, transversely isotropic or anisotropic (Carleton et al. 2014). Understanding the connections between the microscopic structures and the macroscopic functions of fibrous biomaterials is an important topic in tissue engineering. It can advance various biomedical 
applications, such as tissue engineered scaffolds (Stephens et al. 2011, Tseng et al. 2013) and arterial heart valves (Flanagan et al. 2003; Durst et al. 2011; Hasan et al. 2014). Numerical methods, especially the finite element method (FEM), are effective in studying such connections. In literature, there are many efforts to incorporate the biomaterial microscopic structure in the numerical simulation. These efforts can be categorized into two different modeling methodologies.

The first modeling methodology introduces the concept of structural tensor (Spencer 1984) that appears in the total strain energy function to reflect the material microscopic structure. For example, Holzapfel et al. (2000, 2004) included the invariants of two structural tensors to study the influence of fibers that correspond to the collagenous component of the material on the mechanical behavior of arterial wall. Gasser et al. (2006) generalized the concept of structural tensor to consider the influence of fiber dispersions. This modeling methodology usually obtains fiber orientations from a statistical analysis of material histology. The resulting constitutive model can successfully capture the material anisotropy and has important mathematical properties, such as polyconvexity (Schröder and Neff 2002; Itskov and Aksel 2004; Balzani et al. 2006), to guarantee the existence of a deformation field that minimizes the total strain energy. When all the fibers are relatively concentrated in a limited number of orientations, this methodology is very effective to reflect the influence of material microscopic structure, since the fiber orientations can be represented by unit directional vectors. However, when the fibers are highly dispersed inside the material, it is not sufficient to just use structural tensors to depict the fiber network topology. Moreover, this modeling methodology essentially belongs to the homogenization category, assuming that the reinforced-fibers are smeared into the ground matrix material. Therefore, the local stress and strain distributions in different material components cannot be obtained, which limits the information available to describe the connections between the material microscopic structure and macroscopic behavior.

The second methodology relies on directly incorporating the detailed topology of the microscopic fiber network structure into the simulation. The geometry of the fiber network is either synthetically generated (Huisman et al. 2007, Liu et al. 2013, Heidemann et al. 2015) or obtained using image processing from real tissues (D'Amore et al. 2014; Carleton et al. 2014). Depending on the different assumptions of the load transfer mechanism inside the material, the affine or nonaffine fiber kinematics can be used. The affine fiber kinematics assumes that the primary load transfer happens between the fiber and the matrix, and the fiber deformation is completely decided by the macroscopic strain field (Lanir 1983; MacKintosh et al. 1995; Agoram and Barocas 2001: Storm et al. 2005). In contrast, the nonaffine fiber kinematics assumes that the primary load transfer happens among fiber chains and each fiber chain deforms independently of the matrix (Huisman et al. 2007; Stylianopoulos and Barocas 2007; Liu et al. 2013). Several studies have been conducted to compare the influences of the affine and nonaffine kinematics on the material behavior. The predicted material mechanical behavior based on the affine kinematics is stiffer than the one based on the nonaffine kinematics (Chandran and Barocas 2006, Heidemann et al. 2015). In order to bridge the microscopic fiber network structure and the macroscopic material behavior, multiscale simulations can be adopted (Stylianopoulos and Barocas 2007, Chandran et al. 2008), and representative volume elements (RVEs) need to be identified to eliminate the size effect and describe the overall macroscopic material response (Shahsavari and Picu 2013 Carleton et al. 2014, D'Amore et al. 2014). However, all these efforts assume the superposition of the contributions from the fiber network and ground matrix on the global material properties, thus preclude the influence of fiber-matrix interaction. To address this limitation, Lake et al. (2012) developed a two-component microscale model to study the interaction between collagen and matrix material in collagen-agarose co-gels under uniaxial tension. Zhang et al. (2013b) proposed a coupled fiber-matrix model to quantify the influence of fiber network on the stress distribution within the soft tissue. In these works, special finite element mesh generation techniques are needed, and fibers are modeled as truss or beam elements. Therefore, the number of degrees of freedom (DOFs) in the FE simulation increases with the number of fiber segments contained in the network. When a network containing a large number of fiber segments is adopted in the simulation and a comprehensive parametric study is needed, the computational cost is prohibitively expensive.

This paper presents an efficient computational framework that goes midway between homogenizing the fiber network into the ground matrix and accounting for the fully coupled fiber-matrix interaction at the segment length scale. The microscopic fiber network is directly incorporated into the FE simulation without significantly increasing the computational cost. The embedded fiber approach is adopted, which is essentially equivalent to the affine fiber kinematics. The fiber-matrix interaction is partially considered in the sense that the two material components deform together. Monte Carlo (MC) simulations can be easily combined with the 
proposed method to reflect the influence of fiber network randomness on the material mechanical behavior. The proposed computational framework can be used to study the connection between the microscopic structure and macro-mechanical behavior of fibrous biomaterials with a reasonable computational cost. The paper is organized as follows. In Sect. 2, the random walk algorithm implemented to generate the 2D fiber network is described, and a data structure is proposed to record the network geometric information. In Sect. 3. based on the embedded fiber assumption and the fiber strain energy function, a variational approach is adopted to derive the element residual and stiffness matrices for nonlinear FE simulation. In Sect. 4, several numerical examples are provided to demonstrate the accuracy and the predictive capacities of the proposed framework. In Sect. 5. conclusions and limitations of this work are discussed.

\section{Random fiber network structure}

The fibrous biomaterial can be modeled with two basic components, the ground matrix and the network composed by multiple reinforced-fibers. The literature describes several ways to generate the fiber network structure, either in 2D (Spanos and Esteva 2009, Carleton et al. 2014) or 3D (Huisman et al. 2007, Stylianopoulos and Barocas 2007; Zhang et al. 2013b). Here, a random walk algorithm proposed by Spanos and Esteva (2009) is implemented to generate the synthetic fiber network structure in 2D for simulation purposes. The generated fiber network contains multiple fiber chains that are interconnected, and each fiber chain is piecewise linear and composed by multiple fiber segments. The current implementation is based on the following assumptions: 1. The total number of fiber chains contained in the network is decided by the fiber volume fraction $\mu$;

2. Each fiber chain is formed by a fixed number of fiber segments $N_{\text {seg }}$, and each fiber segment has length $l_{\text {seg }}$; 3. The relative angle $\theta$ between two neighboring fiber segments in the same fiber chain follows the uniform distribution, $\theta \sim \operatorname{unif}\left(-\theta_{\max }, \theta_{\max }\right)$.

The main steps of the algorithm are summarized as follows:

1. Specify a 2D area $A$ with the thickness assumed equal to the fiber cross-section diameter $d$;

2. Generate a random point inside $A$ as the starting point of a fiber chain;

3. Use the starting point and a random angle $\theta_{0} \sim$ unif $(-2 \pi, 2 \pi)$ to form the first fiber segment with length $l_{\text {seg }}$;

4. Generate the next connected fiber segment with the angle $\theta \sim \operatorname{unif}\left(-\theta_{\max }, \theta_{\max }\right)$ relative to the previous segment;

5. Repeat Step 4 until the fiber chain is out of the boundary of $A$ or the maximum segment number $N_{\text {seg }}$ in the fiber chain is reached;

6. Repeat Steps 2 to 5 until the assigned fiber volume fraction $\mu$ is reached.

7. Calculate the coordinates of the intersection points formed by all the fiber chains. These intersection points are treated as crosslinks to connect individual fiber chains.

The algorithm has a total of five control parameters $\mu, d, N_{\text {seg }}, l_{\text {seg }}$ and $\theta_{\max }$, through which the topology of the generated fiber network (such as fiber density and tortuosity) can be adjusted. A typical fiber network structure generated by the random algorithm is shown in Fig. 1. The crosslinks located on each fiber segment divide it into multiple sub-segments. Crosslinks that are close to each other (distance $<0.1 l_{\text {seg }}$ ) are merged.

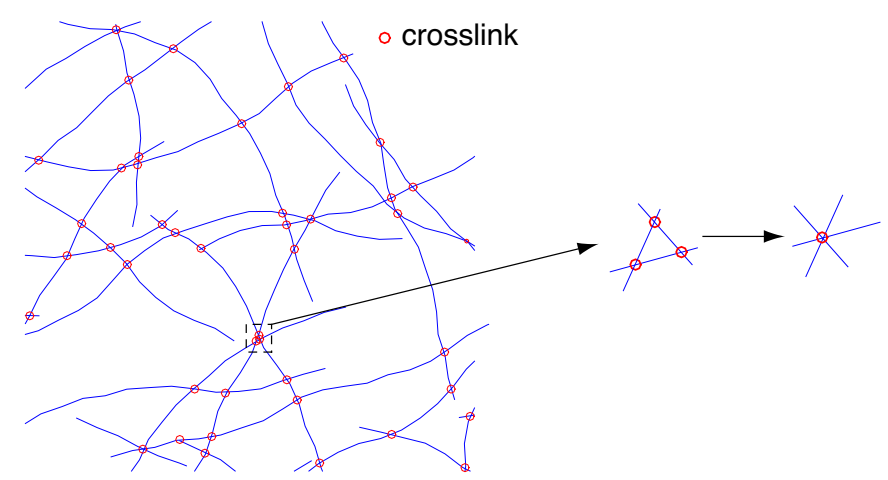

Fig. 1 Fiber network structure generated by the random walk algorithm (crosslinks are formed to connect fiber chains)

The embedded fiber approach is adopted to incorporate the generated fiber network in the simulation. This approach assumes that each fiber chain is completely embedded inside the surrounding ground matrix material, and they deform together. The embedded fiber approach is essentially equivalent to the affine fiber kinematics. Fig. 2 shows a rectangular sample with a fiber network. After the material sample is meshed, each fiber segment is either completely inside one element, or crosses element boundaries. For the latter scenario, the coordinates of the intersection points formed by the segment and the element boundaries are calculated and divide the segment into multiple sub-segments (Fig. 3). The coordinates of the intersection points between fiber segments and element boundaries, crosslinks, and the two endpoints of each fiber segment are collected in a data list $\mathrm{L}=\left\{\left(x_{i}, y_{i}\right), i=1,2,3, \cdots\right\}$. 


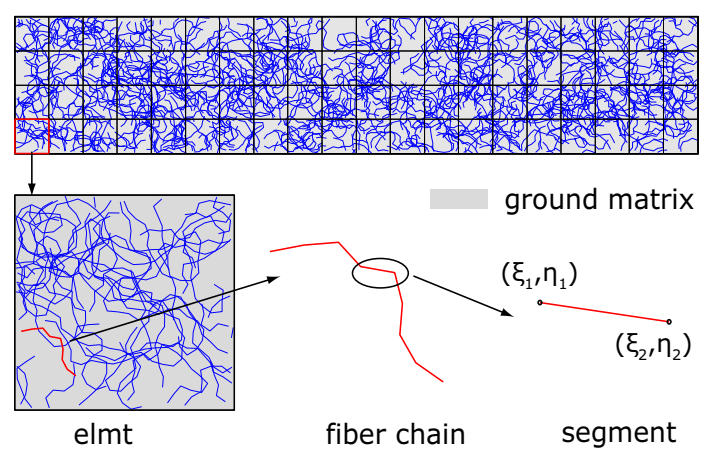

Fig. 2 Fibrous material sample meshed with 4-node quad elements (the fiber network is embedded in the ground matrix material)

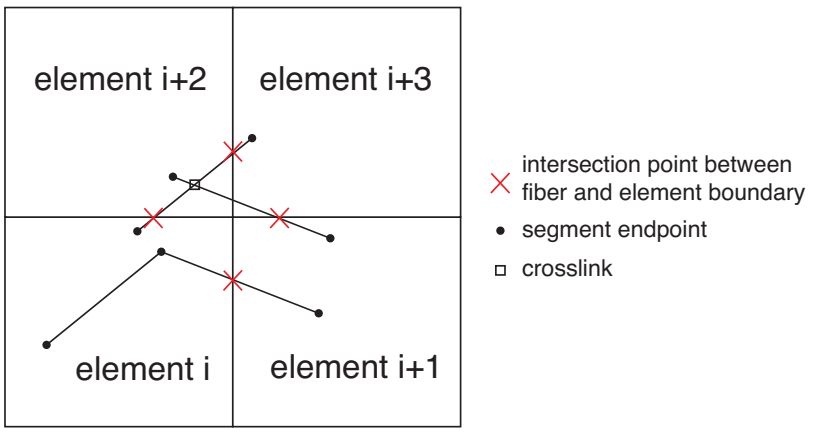

Fig. 3 Intersection points between fiber segments and element boundaries are generated when fiber segments cross element boundaries

In order to include the fiber network structure in the FE simulation, the isoparametric element is used. The natural coordinates $\boldsymbol{\xi}$ of any point in the parent domain and its corresponding Cartesian coordinates $\boldsymbol{x}$ in the current domain have the following isoparametric relationship

$\boldsymbol{x}(\boldsymbol{\xi})=N_{a}(\boldsymbol{\xi}) \boldsymbol{x}_{a}^{(e)}$,

where the subscript $a$ is the element nodal number, $\boldsymbol{x}_{a}^{(e)}$ is the vector of element nodal coordinates for the $e$-th element, and $N_{a}$ is the element interpolation function associated with node $a$. The Einstein summation convention is used. Notice that inside the $e$-th element, the Cartesian coordinates $\boldsymbol{x}$ of the two endpoints of each fiber segment can be obtained from the described data list L. Through Newton-Raphson iterations the natural coordinates $\boldsymbol{\xi}$ corresponding to the Cartesian coordinates $\boldsymbol{x}$ in the list L can be calculated. Following this approach, a data structure (Tab. 1) is constructed. The $i$-th row of this data structure records the natural coordinates of the start $\left(\xi_{1}, \eta_{1}\right)$ and the end $\left(\xi_{2}, \eta_{2}\right)$ of each fiber segment contained in the $i$-th element. Note that different elements do not necessarily have the same number of fiber segments. This data structure will be used in the variational approach to derive the element residual and stiffness matrices.

\section{Variational approach}

Based on the fiber network generated by the random walk algorithm and the embedded fiber assumption, a variational approach is applied to derive the element residual and stiffness matrices. The notation used here has the following convention. Uppercase letters and indices, such as $\boldsymbol{X}, I$ and $J$, represent the variables and their components in the initial (reference) configuration, and lowercase letters and indices, such as $\boldsymbol{x}, i$ and $j$, represent the variables and their components in the current (deformed) configuration.

In the displacement formulation, the primary unknowns are the element nodal displacements $\boldsymbol{u}$. For fibrous materials in which the fiber network is embedded inside the ground matrix material, the total strain energy is expressed as follows

$\Pi(\boldsymbol{u})=\int_{\Omega_{0}} \Psi_{m}(\boldsymbol{F}(\boldsymbol{u})) \mathrm{d} \Omega+\sum_{i=1}^{N} \Psi_{f}\left(\lambda^{(i)}(\boldsymbol{u})\right)$.

In Eq. 2, $\boldsymbol{F}$ is the deformation gradient tensor, $\Psi_{\mathrm{m}}$ is the strain energy function representing the contribution from the ground matrix material, $\Psi_{f}\left(\lambda^{(i)}\right)$ is the strain energy function representing the contribution from the $i$-th segment contained in the fiber network, $N$ is the total number of fiber segments contained in the fiber network, $\Omega_{0}$ is the reference domain, and $\lambda^{(i)}$ is the stretch ratio of the $i$-th fiber segment, defined as

$\lambda^{(i)}=\frac{l^{(i)}}{L_{0}^{(i)}}$,

where $l^{(i)}$ and $L_{0}^{(i)}$ are the deformed and original lengths of the $i$-th fiber segment, respectively.

After the spatial discretization, the total strain energy becomes

$\Pi(\boldsymbol{u})=\sum_{e=1}^{N_{e l}}\left[\int_{\Omega_{0}^{(e)}} \Psi_{m}(\boldsymbol{F}(\boldsymbol{u})) \mathrm{d} \Omega+\sum_{i=1}^{N^{(e)}} \Psi_{f}\left(\lambda^{(i)}(\boldsymbol{u})\right)\right]$

where $N_{e l}$ is the total number of finite elements in the discretized system, $\Omega_{0}^{(e)}$ is the spatial domain occupied by the $e$-th element in the reference configuration, and $N^{(e)}$ is the number of fiber segments contained inside the $e$-th element. 
Table 1 Data structure constructed to record the natural coordinates of the fiber segments contained inside each finite element

\begin{tabular}{c|cccc}
\hline Elmt No. & $\left(\xi_{1}, \eta_{1}\right),\left(\xi_{2}, \eta_{2}\right)$ & $\left(\xi_{1}, \eta_{1}\right),\left(\xi_{2}, \eta_{2}\right)$ & $\cdots$ & $\left(\xi_{1}, \eta_{1}\right),\left(\xi_{2}, \eta_{2}\right)$ \\
\hline 1 & 1st segment & 2nd segment & $\cdots$ & last segment \\
\hline 2 & 1st segment & 2nd segment & $\cdots$ & last segment \\
\hline 3 & 1st segment & 2nd segment & $\cdots$ & last segment \\
\hline$\vdots$ & $\vdots$ & $\vdots$ & $\vdots$ & $\vdots$ \\
\hline $\mathrm{n}$ & 1st segment & 2nd segment & $\cdots$ & last segment \\
\hline
\end{tabular}

The ground matrix material is described by the modified neo-Hookean model with the strain energy function

$\Psi_{m}(\boldsymbol{F})=\frac{1}{4} K\left(J^{2}-1-2 \ln J\right)+\frac{1}{2} G\left(\overline{I_{1}}-3\right)$,

where $K$ is the bulk modulus, $G$ is the shear modulus, $J=\operatorname{det} \boldsymbol{F}$ is the determinant of the deformation gradient tensor $\boldsymbol{F}$, and $\bar{I}_{1}=J^{-\frac{2}{3}} \operatorname{tr}\left(\boldsymbol{F}^{\mathrm{T}} \boldsymbol{F}\right)$ is the modified tensor invariant. The derivations and expressions of the element residual force $\mathbf{R}_{m}^{(e)}$ and the stiffness matrix $\mathbf{K}_{m}^{(e)}$ based on Eq. 5 can be found in most nonlinear FEM textbooks, for example, see (Holzapfel 2000; Bonet and Wood 2008).

The fiber segment embedded in the ground matrix material is modeled as a truss element. Since it is a widely accepted assumption that wavy fibers can only sustain tensile force, but are not able to resist compression (Holzapfel et al. 2000; Balzani et al. 2006; Pandolfi and Vasta 2012), the contribution from each fiber segment is switched off if its stretch ratio $\lambda<1$, indicating a compressive status. In this paper, two different forms of fiber strain energy function $\Psi_{f}$ are used as examples, including the quadratic form

$\Psi_{f}(\lambda)=\left\{\begin{array}{cc}\frac{1}{2} k L_{0}^{2}(\lambda-1)^{2} & \lambda \geq 1 \\ 0 & \lambda<1,\end{array}\right.$

and the exponential form

$\Psi_{f}(\lambda)=\left\{\begin{array}{cc}\frac{1}{2 b} k L_{0}^{2}\left(e^{b(\lambda-1)^{2}}-1\right) & \lambda \geq 1 \\ 0 & \lambda<1,\end{array}\right.$

where $k$ is the axial elastic modulus of the fiber segment at low strains, and $b$ is a dimensionless parameter. A similar exponential form that belongs to the Fung-type model (Fung 1967), is also adopted by Stylianopoulos and Barocas (2007).

If $\Psi_{f}$ takes the exponential form (Eq. 7), for an arbitrary fiber segment with the stretch ratio $\lambda$, the element residual force $\mathbf{R}_{f}^{(e)}$ is obtained (the detailed derivation is shown in Appendix)

$$
\mathbf{R}_{f}^{(e)}=k e^{b(\lambda-1)^{2}}\left(1-\frac{1}{\lambda}\right)\left(\boldsymbol{x}^{(2)}-\boldsymbol{x}^{(1)}\right) \cdot\left(\delta \boldsymbol{u}^{(2)}-\delta \boldsymbol{u}^{(1)}\right)
$$

After linearization the element stiffness matrix $\mathbf{K}_{f}^{(e)}$ is obtained (the detailed derivation is shown in Appendix)

$$
\begin{aligned}
\mathbf{K}_{f}^{(e)}= & k e^{b(\lambda-1)^{2}}\left(1-\frac{1}{\lambda}\right) \\
& \left(\Delta \boldsymbol{u}^{(2)}-\Delta \boldsymbol{u}^{(1)}\right) \cdot\left(\delta \boldsymbol{u}^{(2)}-\delta \boldsymbol{u}^{(1)}\right) \\
+ & \frac{k e^{b(\lambda-1)^{2}}}{L_{0}^{2}}\left(2 b-4 b \lambda^{-1}+2 b \lambda^{-2}+\lambda^{-3}\right) \\
& {\left[\left(\boldsymbol{x}^{(2)}-\boldsymbol{x}^{(1)}\right) \cdot\left(\Delta \boldsymbol{u}^{(2)}-\Delta \boldsymbol{u}^{(1)}\right)\right] } \\
& {\left[\left(\boldsymbol{x}^{(2)}-\boldsymbol{x}^{(1)}\right) \cdot\left(\delta \boldsymbol{u}^{(2)}-\delta \boldsymbol{u}^{(1)}\right)\right] . }
\end{aligned}
$$

In Eqs. 8 and 9, the superscripts (1) and (2) represent the two ends of the fiber segment, and $\boldsymbol{x}^{(i)}, \boldsymbol{u}^{(i)}$ $(i=1,2)$ are the coordinates and displacements of the fiber segment endpoints in the current configuration, respectively.

In the isoparametric element, the fiber kinematics can be expressed by the element nodal displacements via the element interpolation functions, $\bullet^{(i)}=$ $N_{A}\left(\boldsymbol{\xi}^{(i)}\right) \bullet{ }_{A}$, where $\bullet$ can be $\boldsymbol{X}, \boldsymbol{x}$, or $\boldsymbol{u} . \boldsymbol{X}_{A}$ and $\boldsymbol{x}_{A}$ represent the element nodal coordinates in the reference and deformed configurations respectively, $\boldsymbol{u}_{A}$ is the element nodal displacement, the subscript $A$ is the element nodal number, $\boldsymbol{X}^{(i)}$ and $\boldsymbol{x}^{(i)}(i=1,2)$ are the coordinates of the fiber segment endpoints in the reference and deformed configurations, and $\boldsymbol{\xi}^{(i)}$ are the corresponding natural coordinates in the parent domain. With the above isoparametric relationship, the stretch ratio of 
the fiber segment is expressed as follows

$$
\begin{aligned}
\lambda & =\frac{l}{L_{0}} \\
& =\frac{\left\|\boldsymbol{x}^{(2)}-\boldsymbol{x}^{(1)}\right\|_{2}}{\left\|\boldsymbol{X}^{(2)}-\boldsymbol{X}^{(1)}\right\|_{2}} \\
& =\frac{\left\|N_{A}\left(\boldsymbol{\xi}^{(2)}\right) \boldsymbol{X}_{A}-N_{A}\left(\boldsymbol{\xi}^{(1)}\right) \boldsymbol{X}_{A}\right\|_{2}}{\left\|N_{A}\left(\boldsymbol{\xi}^{(2)}\right) \boldsymbol{x}_{A}-N_{A}\left(\boldsymbol{\xi}^{(1)}\right) \boldsymbol{x}_{A}\right\|_{2}} .
\end{aligned}
$$

Combining Eq. 10 with the relationship

$\boldsymbol{x}^{(i)}=\boldsymbol{X}^{(i)}+\boldsymbol{u}^{(i)} \quad(i=1,2)$,

the components of $\mathbf{R}_{f}^{(e)}$ and $\mathbf{K}_{f}^{(e)}$ can be written

$$
\begin{aligned}
& R_{f, i A}^{(e)}= k e^{b(\lambda-1)^{2}}\left(1-\frac{1}{\lambda}\right)\left(x_{i}^{(2)}-x_{i}^{(1)}\right) \\
&\left(N_{A}\left(\boldsymbol{\xi}^{(2)}\right)-N_{A}\left(\boldsymbol{\xi}^{(1)}\right)\right), \\
& K_{f, A i B j}^{(e)}= k e^{b(\lambda-1)^{2}}\left(1-\frac{1}{\lambda}\right) \\
&\left(N_{A}\left(\boldsymbol{\xi}^{(2)}\right)-N_{A}\left(\boldsymbol{\xi}^{(1)}\right)\right) \\
&\left(N_{B}\left(\boldsymbol{\xi}^{(2)}\right)-N_{B}\left(\boldsymbol{\xi}^{(1)}\right)\right) \delta_{i j} \\
&+ \frac{k e^{b(\lambda-1)^{2}}}{L_{0}^{2}}\left(2 b-4 b \lambda^{-1}+2 b \lambda^{-2}+\lambda^{-3}\right) \\
& {\left[\left(x_{i}^{(2)}-x_{i}^{(1)}\right)\left(N_{A}\left(\boldsymbol{\xi}^{(2)}\right)-N_{A}\left(\boldsymbol{\xi}^{(1)}\right)\right)\right] } \\
& {\left[\left(x_{j}^{(2)}-x_{j}^{(1)}\right)\left(N_{B}\left(\boldsymbol{\xi}^{(2)}\right)-N_{B}\left(\boldsymbol{\xi}^{(1)}\right)\right)\right], }
\end{aligned}
$$

where $A, B$ are the element nodal numbers, and $i, j$ are the Cartesian directions. Recall that the natural coordinates $\boldsymbol{\xi}^{(1)}=\left(\xi_{1}, \eta_{1}\right)$ and $\boldsymbol{\xi}^{(2)}=\left(\xi_{2}, \eta_{2}\right)$ of all the fiber segments contained inside each element are available from the data structure shown in Tab. 1. The total element residual $\mathbf{R}^{(e)}$ and the stiffness matrix $\mathbf{K}^{(e)}$ are the sum of the contributions from the ground matrix material and all the fiber segments contained in the corresponding element

$\mathbf{R}^{(e)}=\mathbf{R}_{m}^{(e)}+\sum_{i} \mathbf{R}_{f_{i}}^{(e)}$,

$\mathbf{K}^{(e)}=\mathbf{K}_{m}^{(e)}+\sum_{i} \mathbf{K}_{f_{i}}^{(e)}$.

\section{Numerical examples}

Based on the expressions of the element residual and stiffness matrices derived in Sect. 3 , a user element subroutine is implemented in an open source finite element analysis package FEAP (Taylor 2011). A complete numerical simulation using the proposed framework can be divided into three main parts: preprocessing, FE simulation, and post-processing (Fig. 4).

During preprocessing, firstly the random walk algorithm is applied to generate the fiber network structure with the assigned control parameters. Then, the FE mesh with $N_{e l}$ finite elements is generated, and the intersection points formed between the fiber segments and the element boundaries are calculated. Next, the natural coordinates $\boldsymbol{\xi}$ of all fiber segments inside each element are obtained according to Eq. 1 using the Newton-Raphson algorithm. Lastly, the data structure shown in Tab. 1 is formed. The preprocessing, including the generations of the fiber network and the FE mesh, and the calculations of fiber segment coordinates, is automated in MATLAB ${ }^{\circledR}$.

In the FE simulation, all the fiber segments inside the $e$-th element are looped over to calculate their contributions to the element residual $\mathbf{R}_{f}^{(e)}$ and the stiffness matrix $\mathbf{K}_{f}^{(e)}$. Then according to Eqs. 14 and 15 the contributions from the ground matrix are added to the fiber contributions to form the element residual $\mathbf{R}^{(e)}$ and the stiffness matrix $\mathbf{K}^{(e)}$. Following the same procedure, all the elements $e=1, \ldots, N_{e l}$ are looped over to assemble the global stiffness matrix and the right hand side (RHS). Finally, the discretized problem is solved to obtain the element nodal displacements $\boldsymbol{u}_{A}$.

During post-processing, based on the isoparametric relationship, the deformation and stress distribution in the fiber network and the ground matrix can be obtained separately. The force inside the $i$-th fiber segment $f^{(i)}$ is calculated as follows

$f^{(i)}=\frac{\partial \Psi_{f}\left(\lambda^{(i)}\right)}{L_{0}^{(i)} \partial \lambda^{(i)}}$,

where $\lambda^{(i)}$ and $L_{0}^{(i)}$ refer to the stretch ratio and the original length of the $i$-th fiber segment, respectively.

Following the above procedures, several numerical examples are provided to demonstrate the accuracy and the predictive capability of the proposed computational framework. In the first example, the numerical result is compared with the analytic solution for a rectangular sample embedded with a single fiber under uniform tensile deformation field to show the accuracy of the proposed method. In the second example, a mesh sensitivity analysis is performed on the sample embedded with a random fiber network to examine the influence of the mesh size on the numerical results, such as the force-stretch curve and the stress field fluctuation. In the third example, the proposed method is combined with Monte Carlo (MC) simulations to reflect the network randomness. In the last example, the numerical result is compared with the published experimental data of collagen gels (Roeder et al. 2002) to 
demonstrate the predictive capability of the proposed computational framework.

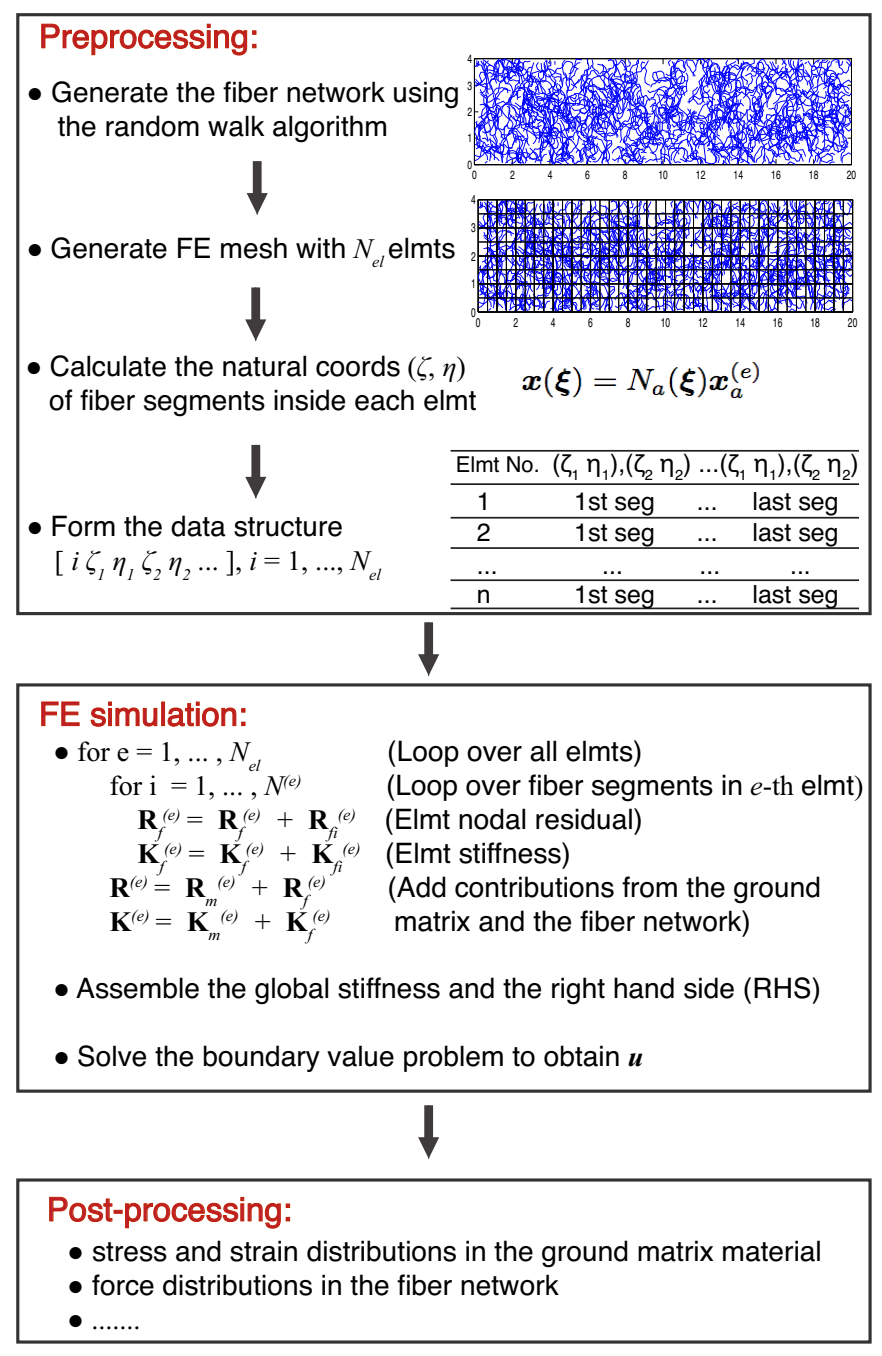

Fig. 4 Flow chart of the proposed computational framework

\subsection{Verification}

In this example, a rectangular sample with the dimension $10 \times 4 \mathrm{~mm}$ has a single fiber embedded. The vertical constraints at the two ends of the sample are chosen to lead to a uniform deformation gradient $\boldsymbol{F}$ (Fig. 5). Also, the ground matrix material is assumed to be incompressible so that

$\boldsymbol{F}=\left(\begin{array}{cc}\lambda & 0 \\ 0 & \frac{1}{\lambda}\end{array}\right)$

where $\lambda$ is the stretch ratio in the 1-direction. The deviatroic part of the strain energy for the matrix mater- ial takes the form of the modified neo-Hookean model (Eq. 5). The Cauchy stress for the matrix material is written as

$\boldsymbol{\sigma}=\boldsymbol{\sigma}_{v o l}+\boldsymbol{\sigma}_{d e v}=p \boldsymbol{I}+G\left(\overline{\boldsymbol{b}}-\frac{1}{2} \overline{I_{1}} \boldsymbol{I}\right)$

where $\mathrm{G}$ is the shear modulus, $p$ is the pressure, $\overline{\boldsymbol{b}}=$ $J^{-\frac{2}{3}} \boldsymbol{b}$ is the modified left Cauchy-Green tensor, and $\bar{I}_{1}=\operatorname{tr}(\overline{\boldsymbol{b}})$. Thus, the Cauchy stress can be expressed in terms of $\lambda$ as follows

$\boldsymbol{\sigma}=G\left(\begin{array}{cc}\frac{1}{2}\left(\lambda^{2}-\lambda^{-2}\right)+p & 0 \\ 0 & \frac{1}{2}\left(\lambda^{-2}-\lambda^{2}\right)+p\end{array}\right)$

Using the free stress boundary condition in the 2direction $\sigma_{22}=0$, the pressure is

$p=\frac{1}{2} G\left(\lambda^{2}-\lambda^{-2}\right)$.

Thus, the matrix stress in the 1-direction results

$\sigma_{11}=G\left(\lambda^{2}-\lambda^{-2}\right)$.

If the fiber strain energy takes the quadratic form (Eq. 6), the total reaction force is

$$
\begin{aligned}
f(\lambda) & =f_{\text {matrix }}+f_{\text {fiber }} \\
& =\sigma_{11} \frac{W_{0}}{\lambda}+k L_{0}(\lambda-1) .
\end{aligned}
$$

If the fiber strain energy takes the exponential form (Eq. 7), the total reaction force is

$$
\begin{aligned}
f(\lambda) & =f_{\text {matrix }}+f_{\text {fiber }} \\
& =\sigma_{11} \frac{W_{0}}{\lambda}+k L_{0}(\lambda-1) e^{(\lambda-1)^{2}} .
\end{aligned}
$$

In the FEM simulation, the matrix material is considered to be nearly incompressible with the Poisson ratio $\nu=0.499$ and Young's modulus $E=0.1 \mathrm{MPa}$. The embedded fiber is assumed to take the quadratic strain energy (Eq. 6) or the exponential strain energy (Eq. 7) with the axial elastic modulus $k=0.01 \mathrm{~N} \cdot \mathrm{mm}^{-1}$. For the exponential strain energy function, $b=2$. The 4 node quadrilateral element with mesh size $h=1 \mathrm{~mm}$ is used, and the FE simulation results are compared with the analytic solutions, as shown in Fig. 6 .

The good matches between the analytical solutions and the numerical results demonstrate the accuracy of the proposed computational framework. Also, from Fig. 6 it can be seen that the sample behavior is largely influenced by the form of the fiber strain energy function. When it takes the quadratic form (Eq. 6), the force-stretch curve softens with the increase of the tensile deformation. When it takes the exponential form (Eq.7), the force-stretch curve stiffens with the increase of the tensile deformation. It is worth emphasizing that 


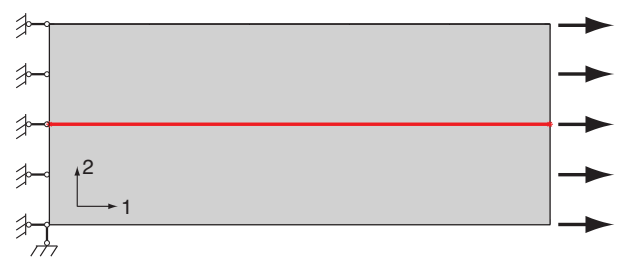

Fig. 5 Rectangular sample embedded with a single fiber under uniaxial tension

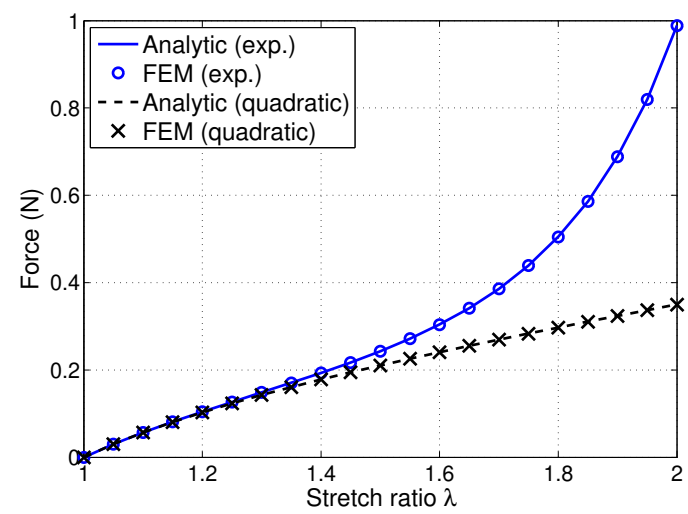

Fig. 6 Comparisons between the analytic solutions and the FEM simulation results using different fiber strain energy functions

the quadratic and the exponential fiber strain energy presented here are just two choices. Depending on the mechanical properties of the fibrous biomaterial of interest, other forms of fiber energy can be used.

\subsection{Mesh sensitivity analysis}

In this example, we consider a rectangular sample (dimension of $20 \times 4 \mathrm{~mm}$ ) with an embedded fiber network. The fiber network is generated by the random walk algorithm with control parameters $\mu=5 \%, N_{\text {seg }}=20$, $l_{\text {seg }}=0.1 \mathrm{~mm}, d=5 \mu \mathrm{m}$ and $\theta_{\max }=30^{\circ}$. The thickness of the sample is assumed equal to the diameter of the fiber segment, and the total length of the generated fiber network is $1018.6 \mathrm{~mm}$. The left end of the sample is fixed in both directions, while the right end is fixed in the 2-direction and displacement control is applied in the 1-direction.

Fig. 7 shows the sample meshed with $50 \times 10$ (the mesh size $h=4 l_{\text {seg }}$ ) 4 -node quadrilateral elements. The natural coordinates of the two endpoints of all the fiber segments in the network, combined with the intersection points formed when the fiber segments cross the element boundaries, are calculated and stored in the data structure during preprocessing. The ground matrix is modeled by the neo-Hookean material law (Eq. 5 ) with Young's modulus $E=0.1 \mathrm{MPa}$ and Poisson ratio $\nu=0.3$. The strain energy of the fiber segments takes the exponential form, as shown in Eq. 7, with $k=0.01$ $\mathrm{N} \cdot \mathrm{mm}^{-1}$ and $b=2.0$.

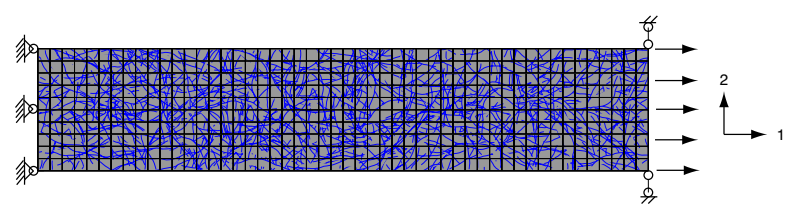

Fig. 7 Rectangular sample embedded with the fiber network meshed by 4-node quad elements with the mesh size $h=4 l_{\text {seg }}$

Recall that when a fiber segment crosses an element boundary, the intersection between this segment and the element boundary divides the segment into two sub-segments that belong to different elements (Fig. 3). Consequently, the discretized system is different when the sample is meshed with different mesh size. To investigate how the mesh size $h$ influences the numerical simulation results, from global measures such as the force-stretch curve, to local measures such as the fluctuations of the matrix stress field and the fiber force distribution, a mesh sensitivity analysis is performed decreasing the mesh size down to the fiber segment length $l_{\text {seg }}$, refinement level where the method is assumed to become accurate.

Fig. 8 shows the influence of mesh refinement on the force-stretch curve of the sample. Fig. 9 shows the influence of the mesh size $h$ on the fluctuation of the matrix stress field and the fiber force distribution inside the network. It can be seen that, while global measures such as the force-stretch curve are not sensitive to the mesh refinement, local measures, such as the matrix stress field and the fiber force distribution, are obviously mesh-dependent. As the mesh size decreases from $h=8 l_{\text {seg }}$ to $h=l_{\text {seg }}$ (the accurate solution is achieved), $\sigma_{11}^{\max }$ in the matrix increases from $86.1 \mathrm{kPa}$ to $124 \mathrm{kPa}$, and $\sigma_{11}^{\min }$ decreases from $69.5 \mathrm{kPa}$ to 50.3 $\mathrm{kPa}$. This increase of the matrix stress field fluctuation (the difference between $\sigma_{11}^{\max }$ and $\sigma_{11}^{\min }$ ) with the mesh refinement is expected, because the element stiffness is directly proportional to the fiber density inside the element. A more refined FE mesh (smaller mesh size $h$ ) makes fiber densities in some elements increase and in other elements decrease. This increase of the fiber density fluctuation due to the mesh refinement leads to the increase of the element stiffness fluctuation, and consequently causes the increase of the maximum matrix stress and the decrease of the minimum matrix stress. 


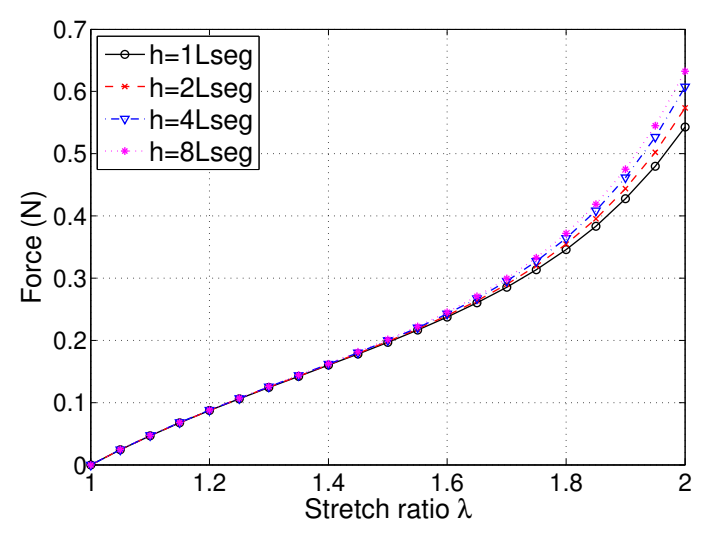

Fig. 8 Influence of mesh size $h$ on the force-stretch curve of the sample under uniaxial tension

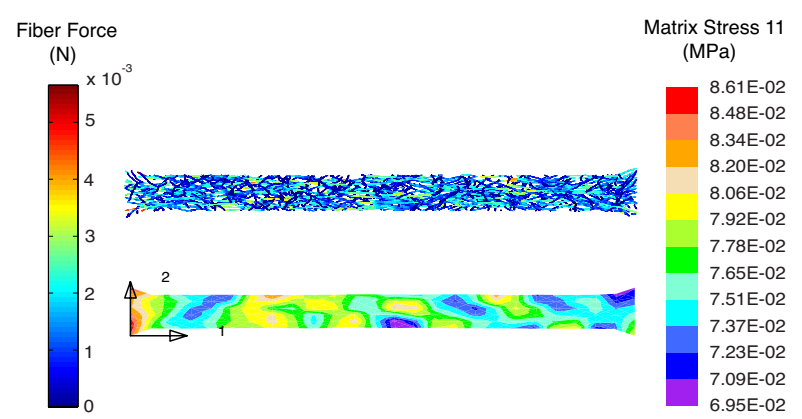

(a) $h=8 l_{\text {seg }}$

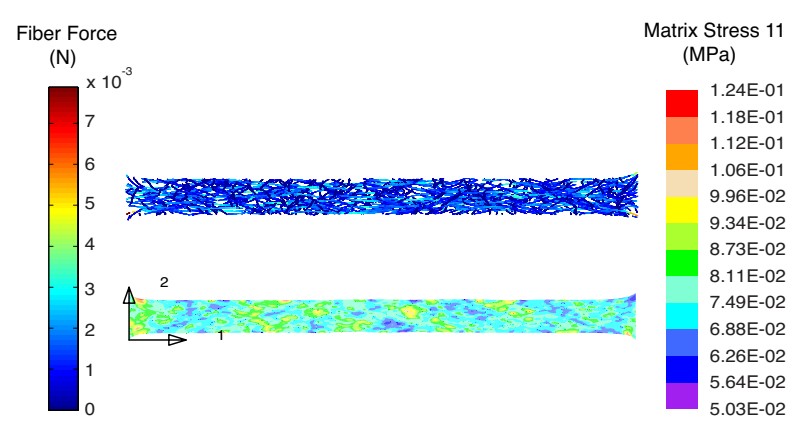

(b) $h=l_{\text {seg }}$

Fig. 9 Influence of mesh size $h$ on the matrix stress field and fiber force distribution when stretch ratio $\lambda=2.0$

In order to quantitatively reflect the influence of the mesh refinement on various numerical results, four different FE mesh sizes $8 l_{\text {seg }}, 4 l_{\text {seg }}, 2 l_{\text {seg }}$ and $l_{\text {seg }}$ are used. For each refinement level, the analysis is performed on five different fiber networks generated from the same random walk algorithm control parameters. All the results are normalized according to the counterparts obtained from the simulation in which the mesh size $h=l_{\text {seg }}$ is used, as shown in Fig. 10. It can be observed that $\sigma_{11}^{\max }$ and $\sigma_{11}^{\min }$ in the matrix increases and decreases with respect to the mesh refinement, re-
Table 2 CPU time

\begin{tabular}{c|cccc}
\hline Mesh size & $l_{\text {seg }}$ & $2 l_{\text {seg }}$ & $4 l_{\text {seg }}$ & $8 l_{\text {seg }}$ \\
\hline CPU time $(\mathrm{s})$ & 24.56 & 4.99 & 1.63 & 0.89 \\
\hline
\end{tabular}

spectively. Global measures such as the initial Young's modulus and the total reaction force are within $10 \%$ error margin even for a coarse mesh $\left(h=8 l_{\text {seg }}\right)$, and the variances of these two quantities are very small case by case so only the average values are reported. Although the CPU time (on a $3.4 \mathrm{GHz}$ Intel Core i7 processor, using OS X 10.8) of the FE simulation increases with the mesh refinement (Tab. 2), it takes only about 25 seconds to finish the simulation when the mesh size $h=l_{\text {seg }}$ is used. The fast simulation speed based on the proposed computational framework is a significant advantage when extensive parametric studies are needed.

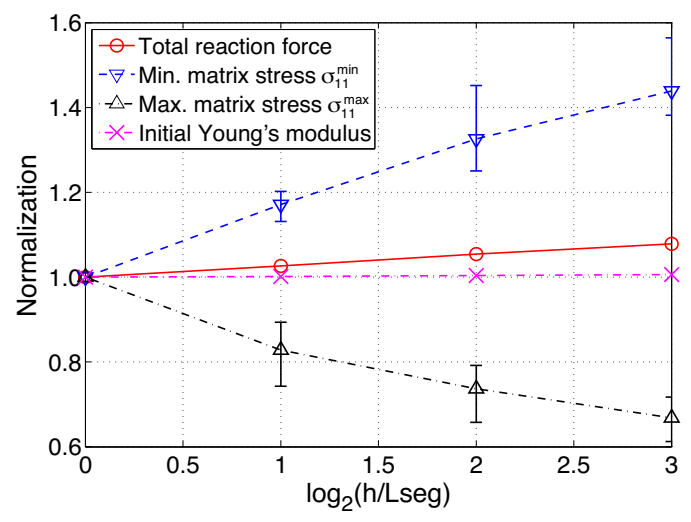

Fig. 10 Influence of mesh size $h$ on various FE simulation results when stretch ratio $\lambda=2.0$ (all the results are normalized according to the counterparts obtained from the case when mesh size $h=l_{\text {seg }}$ is used)

\subsection{Monte Carlo simulation}

In the random walk algorithm used for generating the fiber network, the relative angle $\theta$ between two neighboring fiber segments in the same fiber chain follows the uniform distribution $\theta \sim \operatorname{unif}\left(-\theta_{\max }, \theta_{\max }\right)$. Also, the starting point and the orientation of the first segment in each fiber chain are random. To consider the influence of the stochastic nature of the network on the numerical results, Monte Carlo (MC) simulations are combined with the proposed method to capture the material mechanical behavior in an average sense.

The rectangular sample with the same values of material parameters undergoing uniaxial tension, as shown 
in Fig. 7, is used as an example. Multiple simulations for different network realizations using the same random walk algorithm parameters are performed. Fig. 11 shows the mean and deviation of the force-stretch curves obtained from 500 MC simulations. Fig. 12 shows the convergence of the sample reaction force with respect to the $\mathrm{MC}$ simulations. Although the reaction force varies case by case because of the randomness of the embedded fiber network, it converges after around $300 \mathrm{MC}$ simulations. The total time cost of $500 \mathrm{MC}$ simulations, including the preprocessing and the FE simulation, is approximately one hour.

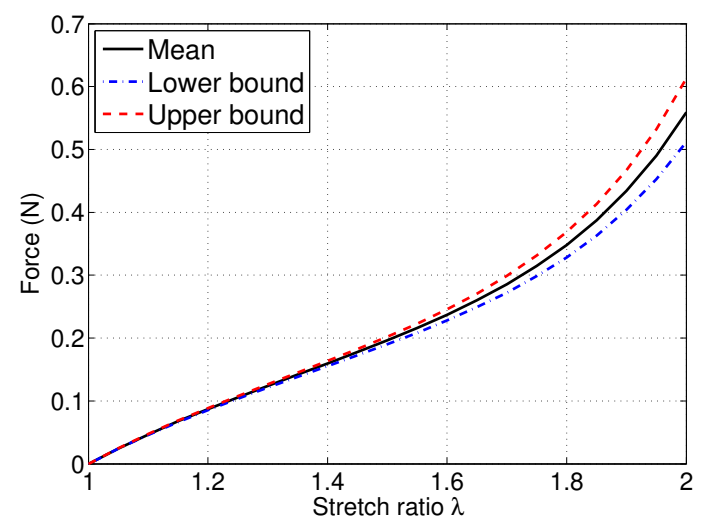

Fig. 11 Mean, lower and upper bounds of the forcestretch curve of the sample obtained from 500 different network realizations with the same values of network parameters

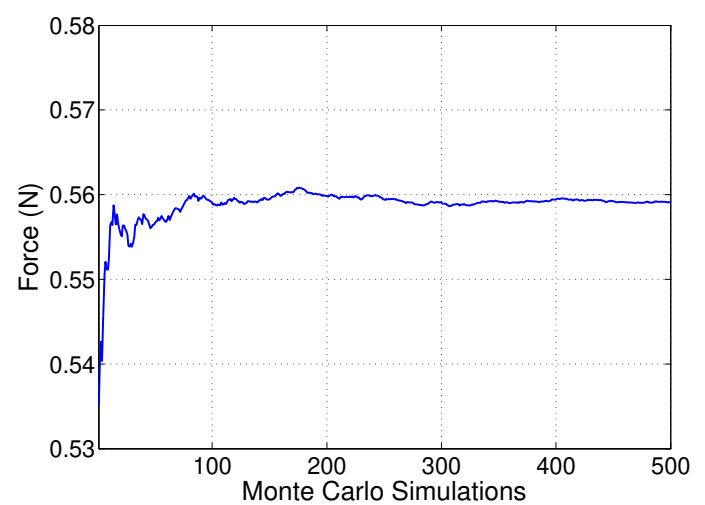

Fig. 12 Convergence of the reaction force with respect to Monte Carlo simulations when the sample is under uniaxial tension and the stretch ratio of the sample $\lambda=$ 2.0

The fiber network structure can be adjusted via the control parameters of the random walk algorithm. The proposed computational framework combined with MC simulation is used to carry out the parametric study of the influence of the embedded fiber network structure on the global material behavior. Fig. 13 shows the influence of the fiber volume fraction $\mu$ on the converged sample reaction force of the MC simulations when the sample is under uniaxial tension and the exponential form Eq. 7 is used as the fiber strain energy. For each value of the fiber volume ratio $\mu, 500$ simulations based on different network realizations are carried out to obtain the mean and deviation of the sample reaction force at the stretch ratio $\lambda=2.0$. The remaining parameters are held constant. The nearly linear relationship between the sample reaction force and the fiber volume fraction is due to the underlying affine fiber kinematics of the proposed method.

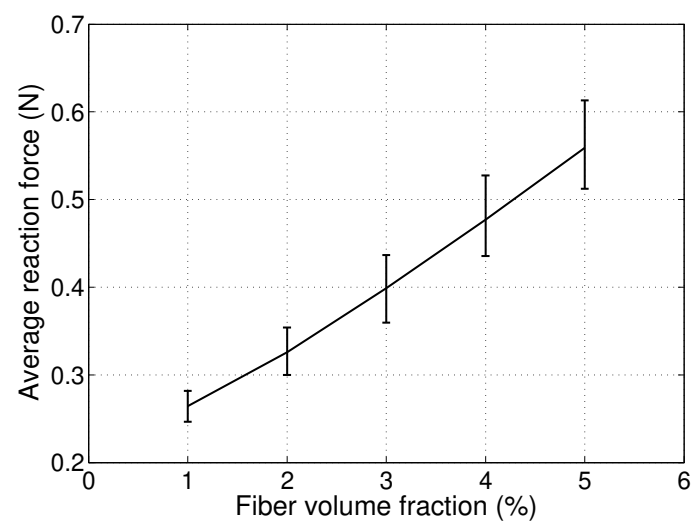

Fig. 13 Influence of the fiber volume fraction $\mu$ on the mean and deviation of the sample reaction force when the stretch ratio $\lambda=2.0$ (obtained from $500 \mathrm{MC}$ simulations)

\subsection{Comparison to published experimental data}

To demonstrate the predictive capability of the proposed computational framework, the numerical results obtained from the FE simulation are compared with the published experimental data of collagen gels under tensile mechanical tests (Roeder et al. 2002). According to the confocal microscopy used in the experimental study, the fibers are randomly oriented, and the mean values of fiber diameter with respect to various collagen concentrations at $\mathrm{pH} 7.4$ are shown in Tab. 3. To convert the collagen concentration to the fiber volume fraction $\mu$, the relationship proposed by Simha et al. (1999) is used

$\mu=1.44 \times($ collagen concentration $)$, 
Table 3 Fiber diameters (mean) of different collagen concentrations reported in (Roeder et al. 2002)

\begin{tabular}{c|cccc}
\hline Collagen concentration $(\mathrm{mg} / \mathrm{ml})$ & 0.3 & 1.0 & 2.0 & 3.0 \\
\hline Mean of fiber diameter $(\mathrm{nm})$ & 418 & 446 & 435 & 430 \\
\hline
\end{tabular}

where the unit of collagen concentration is $\mathrm{g} / \mathrm{cm}^{3}$. For example, the collagen concentration of $2 \mathrm{mg} / \mathrm{ml}$ used in the experiment corresponds to the fiber volume fraction $0.288 \%$.

The original experiment uses a dog-bone shape sample to test the mechanical behavior of collagen gel under uniaxial tension. The middle part of the dog-bone sample undergoes a uniform tensile stretching process. In the FE simulation, only the middle part of the dogbone sample is modeled and a similar boundary condition shown in Fig. 5 is used to mimic the experiment. The modified neo-Hookean material law with Possion ratio $\nu=0.499$ (nearly incompressible) is used to model the ground matrix behavior. The fiber is described by the exponential strain energy function (Eq. 7) with segment length $l_{\text {seg }}=0.1 \mathrm{~mm}$. The material parameters used in the FE simulations are calibrated by fitting the engineering stress-strain curve reported in the experiment, which is available only for the collagen concentration $2 \mathrm{mg} / \mathrm{ml}$. Fig. 14 shows the comparison between the engineering stress reported from the experiment $(2 \mathrm{mg} / \mathrm{ml})$ and the numerical results obtained via 500 network realizations, as well as numerical results for the other three collagen concentrations. The material parameters calibrated from the $2 \mathrm{mg} / \mathrm{ml}$ data include the Young's modulus of the ground matrix $E=1 \mathrm{~Pa}$, the axial elastic modulus $k=2.0 \times 10^{-6} \mathrm{~N} \cdot \mathrm{mm}^{-1}$ and the dimensionless parameter $b=5.5$ of the fiber. The equivalent Young's modulus of fiber based on the above material parameters is $25.1 \mathrm{MPa}$, which is lower than the typically reported range of 0.1 to $10 \mathrm{GPa}$ (Silver et al. 2001). These material parameters and the fiber diameters shown in Tab. 3 are used to calculate the linear moduli of gels with different collagen concentrations (Fig. 15) and the predicted values compare well with the values reported from the experimental data. The nearly linear relationship between the material linear modulus and the gel collagen concentration is expected because of the underlying affine fiber kinematics of the proposed method.

\section{Discussions}

This paper presents a computational framework based on the finite element method (FEM) to simulate the

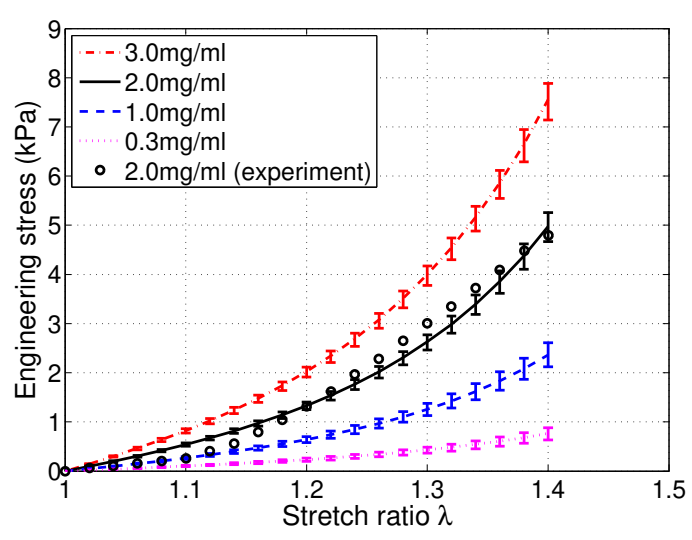

Fig. 14 Comparison of the engineering stress reported from experiment (Roeder et al. 2002) and numerical results obtained via 500 network realizations of different collagen concentrations (the curve of $2.0 \mathrm{mg} / \mathrm{ml}$ is used for material parameter calibration)

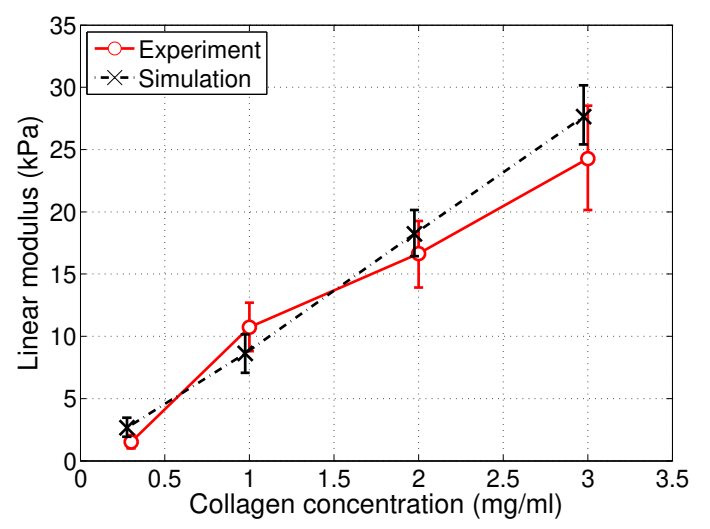

Fig. 15 Comparison of the linear moduli reported from experiment (Roeder et al. 2002) and numerical results obtained via 500 network realizations of different collagen concentrations

mechanical behavior of fibrous materials with randomly distributed fiber networks. The proposed framework adopts a midway modeling methodology between completely homogenizing the fiber network in the ground matrix and considering the fully coupled fiber-matrix interaction at the segment length scale, and can be used to study the connections between the microscopic fiber network structure and the macro-mechanical properties of fibrous biomaterial with a reasonable computational cost.

The proposed method uses the isoparametric relationship to express the fiber kinematics by the element interpolation functions and the element nodal displacements. Therefore, the number of degrees of freedom (DOFs) of the discretized system, which is a direct indicator of computational cost, remains unchanged, even 
if a network containing a large number of fiber segments is incorporated in the FE simulation. The only increase in the computational cost comes from the generation of the fiber network, the calculations of the segment coordinates, and the evaluations of the element interpolation functions at the segment endpoints. The relatively low computational cost of the proposed method can be used to systematically carry out the parametric study of the influence of the fiber network structure on the material macroscopic behavior.

The fibrous biomaterial is typically modeled with two different components, the ground matrix and the fiber network. The proposed method only partially considers the influence of the fiber-matrix interaction in the sense that the two material components deform together, but no relative movement such as slipping and debonding is reflected. This limitation is a direct consequence of using the isoparametric relationship inside the element. Since the fiber network is completely embedded in the surrounding matrix, which is equivalent to the assumption that the primary load transfer inside the material happens between the fiber and the matrix, this approach essentially belongs to the affine fiber kinematics. Both components deform affinely on a scale smaller than the element size. The nearly linear relationship between the fiber volume fraction and the sample reaction force shown in Fig. 13 and the overprediction of the engineering stress at the low strain level (toe region) shown in Fig. 14 are two manifestations caused by the affine network deformation. The nonlinearity of materials with embedded fiber networks comes not only from the mechanical property of individual fiber chains but also from the network effects. A nonaffine fiber kinematics model would predict stronger nonlinearities, even for the quadratic fiber strain energy function.

During the spatial discretization of the fiber network, the intersection points between fiber segment and element boundaries divide the segment into multiple sub-segments. Therefore, the discretized system changes when a different FE mesh size is used. From the mesh sensitivity analysis, it can be seen that although local measures such as stress distribution in the matrix fluctuate according to the mesh size used, the numerical results obtained from different mesh sizes still fall into the same magnitude. This information is still valuable in the sense that the correlation between the local material behaviors and the global mechanical properties can be qualitatively considered. Moreover, global measures such as the initial stiffness and the force-stretch curve of the material are not sensitive to the mesh size. A coarse mesh can be used if the material global mechanical behavior is the only quantity of interest.
It is a widely accepted assumption that fibers can only sustain tensile force, but are unable to resist compression. Based on such assumption, the contribution from a specific fiber segment is switched off if its stretch ratio $\lambda<1$, indicating a compression status. If there is evidence suggesting that fibers in certain biomaterials can also support compressive force, the proposed method can be easily adjusted to meet the observation. In other words, whether fibers are active under compression or not is only a modeling choice depending on the physical properties of the biomaterial of interest (this computational framework handles both). Likewise, the quadratic and the exponential fiber strain energy functions are provided as two examples. In practice, other forms of fiber strain energy can also be adopted. Again, what is the most appropriate strain energy form for fiber depends on the physical properties of the biomaterial of interest.

In this paper, all fiber networks are synthetically generated from the random walk algorithm. The topology of the generated network may not necessarily capture the microscopic structure of biomaterial in reality. A more realistic fiber network can be indirectly generated by the geometric probability based algorithm recently proposed by Carleton et al. (2014), or directly obtained using advanced image processing technologies such as confocal reflection imaging (Roeder et al. 2002, D'Amore et al. 2010, Arganda-Carreras et al. 2010). For example, the segment length $l_{\text {seg }}$ can take the half wavelength of the fiber crimp measured at the microscopic level. The fiber axial elastic modulus can be calculated based on the fiber diameter, the segment length and the fiber Young's modulus in the range reported in literature (Silver et al. 2001). The predicted material macro-mechanical behavior from the numerical simulation largely depends on the number of fibers in the generated network. This parameter can be determined from the fiber volume fraction, fiber diameter and length, if accurate information about the network geometry is available.

In the proposed computational framework, the generated fiber network is random. The stochastic nature of the network influences the numerical simulation results. Consequently, the numerical result from a single simulation would largely depend on the characteristic of the adopted network. To address this disadvantage, multiple simulations based on different network realizations using the same network parameters are needed to capture the material behavior in an average sense. The low computational cost of the proposed method makes it feasible to apply Monte Carlo (MC) simulations to include the randomness of the fiber network structure. 
The convergence (Fig. 12, the mean and deviation of the material behavior (Fig. 11) can be obtained.

The proposed method adopts the affine fiber kinematics, which is shown to be a valid assumption for pericardial collagenous tissues (Fan and Sacks 2014) and mitral valve anterior leaflet (Lee et al. 2015). On the other hand, there is evidence suggesting that the nonaffine fiber kinematics may be a more realistic assumption for certain type of fibrous biomaterial such as bovine annulus fibrous tissue (Head et al. 2003. Chandran and Barocas 2006, Huyghe and Jongeneelen 2012 Lake et al. 2012). How to fully consider the fibermatrix interaction while adopting the nonaffine fiber kinematics in the simulation with a reasonable computational cost remains very challenging and still needs further investigation.

\section{Acknowledgements}

The authors thank Prof. Jane Grande-Allen for useful discussions and suggestions. This work was partially funded by the Ken Kennedy Institute program Collaborative Advances in Biomedical Computing. Funding for this program is made possible by the John \& Ann Doerr Fund for Computational Biomedicine. Their support is greatly appreciated.

\section{Appendix}

For an arbitrary fiber segment contained in an element, use $\boldsymbol{X}^{(1)}$ and $\boldsymbol{X}^{(2)}$ to represent the Cartesian coordinates of its two ends in the reference (initial) configuration. Use $\boldsymbol{x}^{(1)}$ and $\boldsymbol{x}^{(2)}$ to represent the Cartesian coordinates of its two ends in the deformed (current) configuration. Use $\boldsymbol{u}^{(1)}$ and $\boldsymbol{u}^{(2)}$ to represent the displacement of its two ends. The current coordinates of the two ends of the fiber segment can be expressed as

$\boldsymbol{x}^{(i)}=\boldsymbol{X}^{(i)}+\boldsymbol{u}^{(i)}, \quad i=1,2$.

The initial segment length is expressed as

$L_{0}=\left\|\boldsymbol{X}^{(2)}-\boldsymbol{X}^{(1)}\right\|_{2}$,

and the deformed segment length is expressed as

$l=\left\|\boldsymbol{x}^{(2)}-\boldsymbol{x}^{(1)}\right\|_{2}=\left\|\boldsymbol{X}^{(2)}+\boldsymbol{u}^{(2)}-\boldsymbol{X}^{(1)}-\boldsymbol{u}^{(1)}\right\|_{2}$.

Since $\boldsymbol{X}^{(1)}$ and $\boldsymbol{X}^{(2)}$ are constant, the segment stretch ratio is a function of $\boldsymbol{u}^{(1)}$ and $\boldsymbol{u}^{(2)}$

$\lambda=\frac{l}{L_{0}}=\lambda\left(\boldsymbol{u}^{(1)}, \boldsymbol{u}^{(2)}\right)$.
The first variation of the fiber strain energy

$$
\begin{aligned}
& \delta \Psi_{f}\left(\lambda\left(\boldsymbol{u}^{(1)}, \boldsymbol{u}^{(2)}, \delta \boldsymbol{u}^{(1)}, \delta \boldsymbol{u}^{(2)}\right)\right) \\
= & \left.\frac{d}{d \epsilon}\right|_{\epsilon=0} \Psi_{f}\left(\lambda\left(\boldsymbol{u}^{(1)}+\epsilon \delta \boldsymbol{u}^{(1)}, \boldsymbol{u}^{(2)}+\epsilon \delta \boldsymbol{u}^{(2)}\right)\right) \\
= & \left.\frac{\partial \Psi_{f}(\lambda)}{\partial \lambda} \frac{d}{d \epsilon}\right|_{\epsilon=0} \lambda\left(\boldsymbol{u}^{(1)}+\epsilon \delta \boldsymbol{u}^{(1)}, \boldsymbol{u}^{(2)}+\epsilon \delta \boldsymbol{u}^{(2)}\right) .
\end{aligned}
$$

Assume $\Psi_{f}$ take the exponential form Eq. 7 (for other type of fiber strain energy function, the derivation is very similar), then

$$
\begin{aligned}
& \frac{\partial \Psi_{f}(\lambda)}{\partial \lambda}=k L_{0}^{2}(\lambda-1) e^{b(\lambda-1)^{2}}, \\
& \frac{\partial \lambda}{\partial \boldsymbol{u}^{(1)}}=\frac{-1}{L_{0} l}\left(\boldsymbol{X}^{(2)}+\boldsymbol{u}^{(2)}-\boldsymbol{X}^{(1)}-\boldsymbol{u}^{(1)}\right), \\
& \frac{\partial \lambda}{\partial \boldsymbol{u}^{(2)}}=\frac{1}{L_{0} l}\left(\boldsymbol{X}^{(2)}+\boldsymbol{u}^{(2)}-\boldsymbol{X}^{(1)}-\boldsymbol{u}^{(1)}\right),
\end{aligned}
$$

$$
\begin{aligned}
& \left.\frac{d}{d \epsilon}\right|_{\epsilon=0} \lambda\left(\boldsymbol{u}^{(1)}+\epsilon \delta \boldsymbol{u}^{(1)}, \boldsymbol{u}^{(2)}+\epsilon \delta \boldsymbol{u}^{(2)}\right) \\
= & \frac{\partial \lambda}{\partial \boldsymbol{u}^{(1)}} \cdot \delta \boldsymbol{u}^{(1)}+\frac{\partial \lambda}{\partial \boldsymbol{u}^{(2)}} \cdot \delta \boldsymbol{u}^{(2)} \\
= & \frac{1}{L_{0} l}\left(\boldsymbol{X}^{(2)}+\boldsymbol{u}^{(2)}-\boldsymbol{X}^{(1)}-\boldsymbol{u}^{(1)}\right) \cdot\left(\delta \boldsymbol{u}^{(2)}-\delta \boldsymbol{u}^{(1)}\right) \\
= & \frac{1}{L_{0} l}\left(\boldsymbol{x}^{(2)}-\boldsymbol{x}^{(1)}\right) \cdot\left(\delta \boldsymbol{u}^{(2)}-\delta \boldsymbol{u}^{(1)}\right) .
\end{aligned}
$$

Combining Eqs. 29 to 33 , the element residual contributed by the fiber segment under consideration has the form

$$
\begin{aligned}
\mathbf{R}_{f}^{(e)} & =\delta \Psi_{f}\left(\lambda\left(\boldsymbol{u}^{(1)}, \boldsymbol{u}^{(2)}, \delta \boldsymbol{u}^{(1)}, \delta \boldsymbol{u}^{(2)}\right)\right) \\
& =k e^{b(\lambda-1)^{2}}\left(1-\frac{1}{\lambda}\right)\left(\boldsymbol{x}^{(2)}-\boldsymbol{x}^{(1)}\right) \cdot\left(\delta \boldsymbol{u}^{(2)}-\delta \boldsymbol{u}^{(1)}\right) .
\end{aligned}
$$

The linearization of the first variation

$$
\begin{aligned}
& \mathrm{D}_{\Delta \boldsymbol{u}} \delta \Psi_{f}\left(\lambda\left(\boldsymbol{u}^{(1)}, \boldsymbol{u}^{(2)}, \delta \boldsymbol{u}^{(1)}, \delta \boldsymbol{u}^{(2)}\right)\right) \\
= & \left.\frac{d}{d \epsilon}\right|_{\epsilon=0} \delta \Psi_{f}\left(\lambda\left(\boldsymbol{u}^{(1)}+\epsilon \Delta \boldsymbol{u}^{(1)}, \boldsymbol{u}^{(2)}+\epsilon \Delta \boldsymbol{u}^{(2)}\right)\right) \\
= & \left.\frac{d}{d \epsilon}\right|_{\epsilon=0}\left[k e^{b\left(\lambda\left(\boldsymbol{u}^{(1)}+\epsilon \Delta \boldsymbol{u}^{(1)}, \boldsymbol{u}^{(2)}+\epsilon \Delta \boldsymbol{u}^{(2)}\right)-1\right)^{2}}\right. \\
& \left(1-\frac{1}{\lambda\left(\boldsymbol{u}^{(1)}+\epsilon \Delta \boldsymbol{u}^{(1)}, \boldsymbol{u}^{(2)}+\epsilon \Delta \boldsymbol{u}^{(2)}\right)}\right) \\
& \left.\left(\boldsymbol{x}^{(2)}+\epsilon \Delta \boldsymbol{u}^{(2)}-\boldsymbol{x}^{(1)}-\epsilon \Delta \boldsymbol{u}^{(1)}\right) \cdot\left(\delta \boldsymbol{u}^{(2)}-\delta \boldsymbol{u}^{(1)}\right)\right] \\
= & (1)+(2),
\end{aligned}
$$


where

$$
\begin{aligned}
\text { (1) }= & k e^{b(\lambda-1)^{2}}\left(1-\frac{1}{\lambda}\right) \\
& \left(\Delta \boldsymbol{u}^{(2)}-\Delta \boldsymbol{u}^{(1)}\right) \cdot\left(\delta \boldsymbol{u}^{(2)}-\delta \boldsymbol{u}^{(1)}\right), \\
(2)= & \frac{k e^{b(\lambda-1)^{2}}}{L_{0}^{2}}\left(2 b-4 b \lambda^{-1}+2 b \lambda^{-2}+\lambda^{-3}\right) \\
& {\left[\left(\boldsymbol{x}^{(2)}-\boldsymbol{x}^{(1)}\right) \cdot\left(\Delta \boldsymbol{u}^{(2)}-\Delta \boldsymbol{u}^{(1)}\right)\right] } \\
& {\left[\left(\boldsymbol{x}^{(2)}-\boldsymbol{x}^{(1)}\right) \cdot\left(\delta \boldsymbol{u}^{(2)}-\delta \boldsymbol{u}^{(1)}\right)\right] . }
\end{aligned}
$$

Combining Eqs. 35 to 37, the element stiffness matrix contributed by the fiber segment under consideration has the form

$$
\begin{aligned}
\mathbf{K}_{f}^{(e)}= & (1)+(2) \\
= & k e^{b(\lambda-1)^{2}}\left(1-\frac{1}{\lambda}\right) \\
& \left(\Delta \boldsymbol{u}^{(2)}-\Delta \boldsymbol{u}^{(1)}\right) \cdot\left(\delta \boldsymbol{u}^{(2)}-\delta \boldsymbol{u}^{(1)}\right) \\
+ & \frac{k e^{b(\lambda-1)^{2}}}{L_{0}^{2}}\left(2 b-4 b \lambda^{-1}+2 b \lambda^{-2}+\lambda^{-3}\right) \\
& {\left[\left(\boldsymbol{x}^{(2)}-\boldsymbol{x}^{(1)}\right) \cdot\left(\Delta \boldsymbol{u}^{(2)}-\Delta \boldsymbol{u}^{(1)}\right)\right] } \\
& {\left[\left(\boldsymbol{x}^{(2)}-\boldsymbol{x}^{(1)}\right) \cdot\left(\delta \boldsymbol{u}^{(2)}-\delta \boldsymbol{u}^{(1)}\right)\right] . }
\end{aligned}
$$

\section{References}

Agoram B, Barocas VH (2001) Coupled macroscopic and microscopic scale modeling of fibrillar tissues and tissue equivalents. Journal of Biomechanical Engineering 123(4):362-369

Arganda-Carreras I, Fernández-González R, MuñozBarrutia A, Ortiz-De-Solorzano C (2010) 3D reconstruction of histological sections: Application to mammary gland tissue. Microscopy Research and Technique 73(11):1019-1029

Balzani D, Neff P, Schröder J, Holzapfel GA (2006) A polyconvex framework for soft biological tissues. Adjustment to experimental data. International Journal of Solids and Structures 43(20):6052-6070

Bonet J, Wood R (2008) Nonlinear Continuum Mechanics for Finite Element Analysis, 2nd edn. Cambridge

Carleton JB, D'Amore A, Feaver KR, Rodin GJ (2014) Geometric characterization and simulation of planar layered elastomeric fibrous biomaterials. Acta biomaterialia

Chandran PL, Barocas VH (2006) Affine Versus NonAffine Fibril Kinematics in Collagen Networks: Theoretical Studies of Network Behavior. Journal of Biomechanical Engineering 128(2):259-270
Chandran PL, Stylianopoulos T, Barocas VH (2008) Microstructure-based, multiscale modeling for the mechanical behavior of hydrated fiber networks. Multiscale Modeling and Simulation 7(1):22-43

D'Amore A, Stella JA, Wagner WR, Sacks M (2010) Characterization of the complete fiber network topology of planar fibrous tissues and scaffolds. Biomaterials 31(20):5345-5354

D'Amore A, Amoroso N, Gottardi R, Hobson C, Carruthers C, Watkins S, Wagner WR, Sacks M (2014) From single fiber to macro-level mechanics: A structural finite-element model for elastomeric fibrous biomaterials. Journal of the mechanical behavior of biomedical materials 39:146-161

Durst CA, Cuchiara MP, Mansfield EG, West JL, Grande-Allen KJ (2011) Flexural characterization of cell encapsulated PEGDA hydrogels with applications for tissue engineered heart valves. Acta biomaterialia $7(6): 2467-2476$

Fan R, Sacks M (2014) Simulation of planar soft tissues using a structural constitutive model: Finite element implementation and validation. Journal of biomechanics 47(9):2043-2054

Flanagan TC, Pandit A, Taylor P, Jockenhövel S (2003) Living artificial heart valve alternatives: A review. European Cells and Materials 6:28-45

Fung Y (1967) Elasticity of soft tissues in simple elongation. The American journal of physiology 213(6):1532-1544

Gasser TC, Ogden RW, Holzapfel GA (2006) Hyperelastic modelling of arterial layers with distributed collagen fibre orientations. Journal of the Royal Society Interface 3(6):15-35

Hasan A, Ragaert K, Swieszkowski W, Selimović T, Paul A, Camci-Unal G, Mofrad MRK, Khademhosseini A (2014) Biomechanical properties of native and tissue engineered heart valve constructs. Journal of biomechanics 47(9):1949-1963

Head DA, Levine AJ, MacKintosh FC (2003) Deformation of Cross-Linked Semiflexible Polymer Networks. Physical Review Letters 91(10):1081,021-1081,024

Heidemann KM, Sharma A, Rehfeldt F, Schmidt CF, Wardetzky M (2015) Elasticity of 3D networks with rigid filaments and compliant crosslinks. Soft Matter $11(2): 343-354$

Holzapfel GA (2000) Nonlinear Solid Mechanics, a Continuum Approach for Engineering. Wiley

Holzapfel GA, Gasser TC, Ogden RW (2000) A new constitutive framework for arterial wall mechanics and a comparative study of material models. Journal of Elasticity 61(1-3):1-48

Holzapfel GA, Gasser TC, Ogden RW (2004) Comparison of a Multi-Layer Structural Model for Arterial 
Walls with a Fung-Type Model, and Issues of Material Stability. Journal of Biomechanical Engineering 126(2):264-275

Huisman EM, van Dillen T, Onck PR, Van der Giessen E (2007) Three-dimensional cross-linked F-actin networks: Relation between network architecture and mechanical behavior. Physical Review Letters 99(20)

Huyghe JM, Jongeneelen CJM (2012) 3D non-affine finite strains measured in isolated bovine annulus fibrosus tissue samples. Biomechanics and Modeling in Mechanobiology 11(1-2):161-170

Itskov M, Aksel N (2004) A class of orthotropic and transversely isotropic hyperelastic constitutive models based on a polyconvex strain energy function. International Journal of Solids and Structures 41(14):3833-3848

Lake SP, Hadi MF, Lai VK, Barocas VH (2012) Mechanics of a fiber network within a non-fibrillar matrix: Model and comparison with collagen-agarose co-gels. Annals of Biomedical Engineering 40(10):2111-2121

Lanir Y (1983) Constitutive equations for fibrous connective tissues. Journal of biomechanics 16(1):1-12

Lee CH, Zhang W, Liao J, Carruthers CA, Sacks JI, Sacks MS (2015) On the presence of affine fibril and fiber kinematics in the mitral valve anterior leaflet. Biophysical Journal 108(8):2074-2087

Liu Q, Lu Z, Hu Z, Li J (2013) Finite element analysis on tensile behaviour of 3D random fibrous materials: Model description and meso-level approach. Materials Science and Engineering A 587:36-45

MacKintosh FC, Käs J, Janmey PA (1995) Elasticity of semiflexible biopolymer networks. Physical Review Letters 75(24):4425-4428

Pandolfi A, Vasta M (2012) Fiber distributed hyperelastic modeling of biological tissues. Mechanics of Materials 44:151-162

Roeder BA, Kokini K, Sturgis JE, Robinson JP, Voytik-Harbin SL (2002) Tensile mechanical properties of three-dimensional type I collagen extracellular matrices with varied microstructure. Journal of Biomechanical Engineering 124(2):214-222

Schröder J, Neff P (2002) Application of polyconvex anisotropic free energies to soft tissues. In: WCCM V Fifth World Congress on Computational Mechanics July 7-12, 2002, Vienna, Austria

Shahsavari AS, Picu RC (2013) Size effect on mechanical behavior of random fiber networks. International Journal of Solids and Structures 50(20-21):3332-3338

Silver FH, Horvath I, Foran DJ (2001) Viscoelasticity of the vessel wall: The role of collagen and elastic fibers. Critical Reviews in Biomedical Engineering $29(3): 279-301$
Simha NK, Fedewa M, Leo PH, Lewis JL, Oegema T (1999) A composites theory predicts the dependence of stiffness of cartilage culture tissues on collagen volume fraction. Journal of biomechanics 32(5):503509

Spanos P, Esteva M (2009) Effect of stochastic nanotube waviness on the elastic and thermal properties of nanocomposites by fiber embedment in finite elements. Journal of Computational and Theoretical Nanoscience 6(10):2317-2333

Spencer A (ed) (1984) Continuum Theory of the Mechanics of Fiber-reinforced Composites. Springer

Stella JA, Sacks M (2007) On the biaxial mechanical properties of the layers of the aortic valve leaflet. Journal of Biomechanical Engineering 129(5):757766

Stella JA, D'Amore A, Wagner WR, Sacks M (2010) On the biomechanical function of scaffolds for engineering load-bearing soft tissues. Acta biomaterialia $6(7): 2365-2381$

Stephens EH, Durst C, West JL, Grande-Allen K (2011) Mitral valvular interstitial cell responses to substrate stiffness depend on age and anatomic region. Acta biomaterialia $7(1): 75-82$

Storm C, Pastore JJ, MacKintosh FC, Lubensky TC, Janmey PA (2005) Nonlinear elasticity in biological gels. Nature 435(7039):191-194

Stylianopoulos T, Barocas VH (2007) Volumeaveraging theory for the study of the mechanics of collagen networks. Computer Methods in Applied Mechanics and Engineering 196(31-32):2981-2990

Taylor R (2011) FEAP, a Finite Element Analysis Program: Version 8.3 User Manual. University of California, Berkeley. Dept. of Civil and Environmental Engineering

Tseng H, Cuchiara ML, Durst C, Cuchiara MP, Lin CJ, West JL, Grande-Allen K (2013) Fabrication and mechanical evaluation of anatomically-inspired quasilaminate hydrogel structures with layer-specific formulations. Annals of Biomedical Engineering 41(2):398-407

Zhang L, Lake SP, Barocas VH, Shephard MS, Picu RC (2013a) Cross-linked fiber network embedded in an elastic matrix. Soft Matter 9(28):6398-6405

Zhang L, Lake SP, Lai VK, Picu CR, Barocas VH, Shephard MS (2013b) A coupled fibermatrix model demonstrates highly inhomogeneous microstructural interactions in soft tissues under tensile load. Journal of Biomechanical Engineering 135(1):011,008-011,008 